\title{
Partial ptosis, dilated pupils and ataxia following abamectin poisoning
}

\section{H Karunatilake, S Amarasinghe, S Dassanayake, T Saparamadu, S Weerasinghe}

Ceylon Medical Journal 2012; 57: 125-126

\section{Introduction}

Abamectin is a pesticide recently introduced to the local market. Human intoxication with abamectin is not previously reported in Sri Lanka. In contrast to common organophosphate poisoning presented with cholinergic signs patients with abamectin poisoning present with dilated pupils, ataxia and confusion. A patient was seen at our unit following poisoning with abamectin. We present clinical details of the case and follow it with a brief discussion on relevant toxicology.

\section{Case report}

A 30-year old man was transferred from a local hospital with acute onset confusion, blurred vision and unsteady gait. Three hours prior to presentation he had ingested nearly $40 \mathrm{ml}$ of MIG18® (abamectin $18 \mathrm{gm} / \mathrm{l}$ ). He was restless and agitated. He was unable to walk and ataxic on his feet. His Glasgow coma score was 9. He had partial ptosis of both eyes. The pupils were dilated $(3 \mathrm{~mm})$ and were reacting sluggishly to light. There was no fasciculation or excessive sweating. His lung fields were

${ }^{1}$ Medical Unit, General Hospital, Polonnaruwa, Sri Lanka.

Correspondence: HK, e-mail: <sanyalharindra@yahoo.com>. Received 13 September 2011 and revised version accepted 7 January 2012. Competing interests: none declared. 
clear and respiratory rate was 26 breaths $/ \mathrm{min}$. His blood pressure was $120 / 60 \mathrm{mmHg}$ and pulse rate of was $100 / \mathrm{min}$. His tendon reflexes were decreased. Gastric decontamination was done and activated charcoal was given at the local hospital. Patient was observed in the intensive care unit for 24 hours. His serum transaminases, creatinine, urea, amylase and prothrombin time remained within normal limits. He remained agitated for nearly 12 hours and gradually improved. There was no need for inotropic support as fluid replacement was sufficient to maintain the blood pressure. He was sent home with complete recovery.

\section{Discussion}

Abamectin is a mixture of avermectins. Avermectin is a macrocyclic lactone effective against agriculturally important insects and mites [1]. Abamectin is an analog of Ivermectin which has been used in humans against Onchocerca volvulus. The toxic effects of avermectin in humans are not clearly defined. Hence the toxicological data are derived from the patients receiving ivermectin. Reported toxic effects include pruritus, bone pain, fever, hypotension, mydriasis and tachycardia [2]. The only available series which included patients ingested abamectin observed nausea, vomiting, diarrhoea, drowsiness, agitation and weakness with mild poisoning and hypotension, tachycardia, coma and respiratory failure were seen with severe poisoning [3]. Aspiration pneumonia was related to the adverse outcomes in this series.

Abamectin stimulates gama-amino butyric acid (GABA) receptors in central nervous system. Humans are less susceptible to the toxic effects of abamectin as it does not cross the blood brain barrier readily [2]. Abamectin activates the glutamate gated chloride channels in nerve and muscle cells in invertebrates. However, this effect is not observed in humans [4]. Toxic effects of abamectin can be attributed to the GABAergic effects of avermectins.
Treatment of abamectin poisoning is supportive [5]. Protection of the airway is vital. It can prevent aspiration pneumonia. Gastric decontamination and use of activated charcoal is recommended as abamectin is largely excreted via faeces. Use of GABA inhibitor flumazenil had not been useful [3]. Neostigmine has been used successfully in comatose cats with ivermectin toxicity [6]. However, avermectins does not regulate cholinergic transmissions. Picrotoxin, a GABA antagonist has been suggested as an antidote in animals with ivermectin toxicity [4]. GABAergic drugs such as benzodiazepine and barbiturates should be avoided in acute setting. Appropriate fluid resuscitation and inotropic agents may be required in hypotensive patients [5].

\section{References}

1. Lankas G, Gordon L. Toxicology. In: Campbell WC (editor): Ivermectin and Abamectin, 1st Edition. New York: SpringerVerlag, 1989; 131-143.

2. Njoo FL, Beek WM, Keukens HJ, et al. Ivermectin detection in serum of onchocerciasis patients: relationship to adverse reactions. American Journal of Tropical Medicine and Hygiene 1995; 52: 94-7.

3. Chung K, Yang CC, Wu ML, Deng JF, Tsai WJ. Agricultural avermectins: an uncommon but potentially fatal cause of pesticide poisoning. Annals of Emergency Medicine 1999; 34: 51-7.

4. Yang CC. Mectins poisoning vs avermectin poisoning. Proceedings of 7th International Congress of Asia Pacific Association of Medical Toxicology, Taiwan. December 1113, 2007.

5. Soyuncu S, Oktay C, Berk Y, Eken C. Abamectin intoxication with coma and hypotension. Clinical Toxicology 2007; 45: 299-300.

6. Muhammad G, Abdul J, Khan MZ, Saqib M. Use of neostigmine in massive ivermectin toxicity in cats. Veterinary and Human Toxicology 2004; 46: 28-9. 